\title{
Collective versus local measurements on two parallel or antiparallel spins
}

\author{
S. Massar \\ Service de Physique Théorique, Université Libre de Bruxelles, CP 225, Bvd du Triomphe, B1050 Bruxelles, Belgium
}

(November 9, 2018)

\begin{abstract}
We give a complete analysis of covariant measurements on two spins. We consider the cases of two parallel and two antiparallel spins, and we consider both collective measurements on the two spins, and measurements which require only Local Quantum Operations and Classical Communication (LOCC). In all cases we obtain the optimal measurements for arbitrary fidelities. In particular we show that if the aim is determine as well as possible the direction in which the spins are pointing, it is best to carry out measurements on antiparallel spins (as already shown by Gisin and Popescu), second best to carry out measurements on parallel spins and worst to be restricted to LOCC measurements. If the the aim is to determine as well as possible a direction orthogonal to that in which the spins are pointing, it is best to carry out measurements on parallel spins, whereas measurements on antiparallel spins and LOCC measurements are both less good but equivalent.
\end{abstract}

One of the central problems of quantum measurements is how to best estimate the state of an unknown quantum system. This problem has been addressed by many authors, using many different approaches, see [1] [2] for reviews.

In the present paper we take a new look at a particular example in which the task is to determine the direction of polarization of two identical spin $1 / 2$ particles. We suppose that the polarization direction is completely unknown, ie. is uniformly distributed on the sphere. This problem, generalized to the case of an arbitrary number $\mathrm{N}$ of spin $1 / 2$ has already been studied by several authors [2 5]. The particular case of 2 spins has the advantage of being sufficiently simple that a complete solution can be obtained. Furthermore it allows for several twists where particular features of quantum mechanics related to entanglement reveal themselves.

The first twist on the original problem was suggested by Peres and Wootters [6] who asked whether there is a difference between collective, as compared to local, measurements on two particles. Technically, in the first case one allows arbitrary quantum operations on both spins, whereas in the second case one restricts oneself to Local Quantum Operations on each particle and Classical Communication between the particles (LOCC).

Peres and Wootters gave numerical evidence that even if two particles are in the same state, collective measurements can be better than measurements using LOCC. An analytical proof was given in [3] in the case of two identical spin $1 / 2$ whose polarization direction is uniformly distributed on the sphere, at least in the case where there are only a finite number of rounds of communication be- tween the two parties. A remarkable example was exhibited in [7] which consists of a basis of separable states, ie. states that can be prepared using LOCC, but which nevertheless cannot be distinguished unambiguously using LOCC. In [7] the phrase "non locality without entanglement" was coined for this property. Another result is that of [8] where it was shown that in the limit of an infinite number of identical spin $1 / 2$, LOCC measurements perform as well as collective measurements if the spins are in a pure state, but not as well if the spins are in a mixed state.

A second twist on the original scenario was recently proposed by Gisin and Popescu [10] who considered the case of two antiparallel spins. They showed that one can determine better the direction of polarization of two antiparallel spins than of two parallel spins. Gisin and Popescu's result is related to "non locality without entanglement" because if LOCC measurements are carried out on the two spins, it does not make any difference whether the spins are parallel or antiparallel. Thus collective measurements on two antiparallel spins is an example of non locality without entanglement. Mathematically the passage from two parallel to two antiparallel spins, that is the flip of one of the spins, is the same operation that Peres used to distinguish whether two states are entangled or not [9].

The present paper aims at providing an exhaustive analysis of measurements on two spins $1 / 2$ particles in the three cases of collective measurements on parallel spins, collective measurements on antiparallel spins, and LOCC measurements. The spin flip operation will play a central role in this analysis because it will allow us to treat all three cases in the same framework. Our analysis allows one to find the optimal measurements for arbitrary fidelities. As an illustration we consider two such fidelities.

The first fidelity is $f=(1+\cos \theta) / 2$ where $\theta$ is the angle between the direction in which the spins are polarized and the direction in which one guesses that they are polarized. In this case we recover the results of [2] [3] that if the spins are parallel the maximal average fidelity is $f=0.75$. If the spins are antiparallel we show that the maximal fidelity is $f=0.788$. This fidelity was already obtained in [10] but it was not known whether it is optimal. Finally we shall show that if one restricts oneself to LOCC measurements, then the maximal fidelity is $f=0.736$, which is $1.4 \%$ lower than for measurements on parallel spins. That this is the optimal value for LOCC measurements was already found by D.G. Fischer, S.H. Kienle, and M. Freyberger [1]. Thus even in the limit of 
an infinite number of rounds of communication collective measurements are better than LOCC measurements in the case of two parallel spins.

The second fidelity is $f=1-\cos ^{2} \theta$. In this case it is most advantageous to guess a direction orthogonal to that in which the spins are pointing $(\theta=\pi / 2)$ and most disadvantageous to guess in the direction in which the spins are pointing $(\theta=0)$ or in the orthogonal direction $(\theta=\pi)$. Geometrically this can be rephrased as a situation in which the spins encode the orientation of a plane by pointing in the direction normal to the plane and the aim is to find a vector lying in the plane. In this case the highest fidelity $f=0.8$ is obtained when the spins are parallel. Antiparallel spins or LOCC measurements both give the same optimal fidelity $f=0.733$.

We now turn to the proof of these results. Essential to our analysis will be the spin flip operation which we denote by ${ }^{\sim}$. For a single spin $1 / 2$ it takes the form

$$
\rho=\frac{I}{2}+\vec{\alpha} \cdot \vec{\sigma} \quad \rightarrow \quad \rho^{\sim}=\frac{I}{2}-\vec{\alpha} \cdot \vec{\sigma}
$$

where $I$ is the identity operator and $\sigma_{i}$ the Pauli spin operators. In the case of two spins, we will be interested in the operation, denoted ${ }^{2}$ which flips only the second spin. If we write the state as

$$
\rho=\frac{I}{4}+\vec{\alpha} \cdot \vec{\sigma} \otimes \frac{I}{2}+\vec{\beta} \cdot \frac{I}{2} \otimes \vec{\sigma}+\sum_{i, j} \gamma_{i j} \sigma_{i} \otimes \sigma_{j},
$$

then $\rho^{2}$ is given by

$$
\rho^{2}=\frac{I}{4}+\vec{\alpha} \cdot \vec{\sigma} \otimes \frac{I}{2}-\vec{\beta} \cdot \frac{I}{2} \otimes \vec{\sigma}-\sum_{i, j} \gamma_{i j} \sigma_{i} \otimes \sigma_{j}
$$

The ${ }^{2}$ operation is equivalent, up to a unitary operation acting on particle 2 only, to the partial transpose introduced in 9 .

As an application of the ${ }^{2}$ operation consider the state of two parallel spin $1 / 2$ particles both pointing in the $\vec{m}$ direction

$$
\begin{aligned}
\rho(\vec{m}, \vec{m}) & =\left|\uparrow_{\vec{m}}\right\rangle\left\langle\uparrow_{\vec{m}}|\otimes| \uparrow_{\vec{m}}\right\rangle\left\langle\uparrow_{\vec{m}}\right| \\
& =\frac{I}{4}+\sum_{i} m_{i}\left(\sigma_{i} \otimes \frac{I}{2}+\frac{I}{2} \otimes \sigma_{i}\right)+\sum_{i, j} m_{i} m_{j} \sigma_{i} \otimes \sigma_{j}
\end{aligned}
$$

and the state of two antiparallel spins

$$
\rho(\vec{m},-\vec{m})=\left|\uparrow_{\vec{m}}\right\rangle\left\langle\uparrow_{\vec{m}}|\otimes| \uparrow-\vec{m}\right\rangle\left\langle\uparrow_{-\vec{m}}\right| .
$$

We have the relation

$$
\rho(\vec{m}, \vec{m})=\rho(\vec{m},-\vec{m})^{2} .
$$

We can also consider the dual of the ${ }^{2}$ operation, that is how it acts on operators. Suppose that $\rho$ is a state and $a$ an operator, then $a^{2}$ is defined by the relation

$$
\operatorname{Tr} a \rho^{2}=\operatorname{Tr} a^{\sim 2} \rho .
$$

One finds that it takes exactly the same form for operators as it does for states. If the operator $a$ is expressed as

$$
a=w I+\vec{x} \cdot \vec{\sigma} \otimes I+\vec{y} \cdot I \otimes \vec{\sigma}+\sum_{i, j} z_{i j} \sigma_{i} \otimes \sigma_{j},
$$

then the operator $a^{2}$ takes the form

$$
a^{2}=w I+\vec{x} \cdot \vec{\sigma} \otimes I-\vec{y} \cdot I \otimes \vec{\sigma}-\sum_{i, j} z_{i j} \sigma_{i} \otimes \sigma_{j} .
$$

The ${ }^{\sim 2}$ operation for operators allows us to put a restriction on the Positive Operator Valued Measures (POVM) acting on the space of two spin $1 / 2$ particles that can be realized by local Quantum Operations and Classical Communication (LOCC). Indeed it was shown in [7] that such a POVM, defined by its elements $a_{i} \geq 0$, $\sum_{i} a_{i}=1$, must obey $a_{i}{ }^{2} \geq 0$ for all $i$.

To proceed with the proof, consider a set of operators $a_{i}$ that sum to the identity $\sum_{i} a_{i}=1$. We are interested in the following 3 positivity conditions on $a_{i}$ :

1. $a_{i} \geq 0$. In this case the $a_{i}$ constitute a POVM. The probability of getting outcome $i$ if the state is $\rho(\vec{m}, \vec{m})$ is $P_{\|}(i \mid \vec{m})=\operatorname{Tr} \rho(\vec{m}, \vec{m}) a_{i}$.

2. ${a_{i}}^{2} \geq 0$. In this case the $a_{i}^{2}$ constitute a POVM. The probability of getting outcome $i$ if the state is $\rho(\vec{m},-\vec{m})$ is $P_{\perp}(i \mid \vec{m})=\operatorname{Tr} \rho(\vec{m},-\vec{m}){a_{i}}^{2}$. Using equation (17) we have $P_{\perp}(i \mid \vec{m})=\operatorname{Tr} \rho(\vec{m}, \vec{m}) a_{i}$.

3. $a_{i} \geq 0$ and $a_{i}{ }^{2} \geq 0$. In this case both $a_{i}$ and ${a_{i}}^{2}$ constitute a measurement which can be realized by LOCC. The probability $P_{\|}(i \mid \vec{m})=\operatorname{Tr} \rho(\vec{m}, \vec{m}) a_{i}$ of obtaining outcome $i$ if the spins are parallel and the measurement is $a_{i}$ equals the probability $P_{\perp}(i \mid \vec{m})=\operatorname{Tr} \rho(\vec{m}, \overrightarrow{-} m){a_{i}}^{2}$ of of obtaining outcome $i$ if the spins are antiparallel and the measurement is ${a_{i}}^{2}$. The equality of $P_{\|}(i \mid \vec{m})$ and $P_{\perp}(i \mid \vec{m})$ shows that in this case there is no difference between making measurements on parallel and antiparallel spins.

Thus the ${ }^{2}$ operation relates measurements on parallel spins (given by $P_{\|}(i \mid \vec{m})$ ), measurements on antiparallel spins (given by $P_{\perp}(i \mid \vec{m})$ ), and measurements that can be realized by LOCC. The central idea is that by using the ${ }^{\sim 2}$ operation all these quantities can be expressed in terms of the same trace $\operatorname{Tr} \rho(\vec{m}, \vec{m}) a_{i}$, but with operators $a_{i}$ which obey the different positivity conditions enumerated above.

To further explicitise these different positivity conditions we shall suppose that the aim of the measurement is to distinguish along which direction the spins are pointing. We can then label the POVM elements $a_{\vec{n}}$ by the direction $\vec{n}$ along which one guesses the spins are pointing. 
Furthermore we shall suppose that the spins are polarized in a random direction uniformly distributed on the sphere. We can then, without loss of generality [2], suppose that we are dealing with covariant measurements, that is measurements for which the guessed direction $\vec{n}$ spans the whole sphere and which satisfy

$$
\operatorname{Tra}_{\vec{n}} \rho(\vec{m}, \vec{m})=\operatorname{Tra}_{R(\vec{n})} \rho(R(\vec{m}), R(\vec{m}))
$$

where $R$ is an arbitrary rotation, ie. an element of $S O(3)$. Using the fact that $\rho(R(\vec{m}), R(\vec{m}))=R \otimes R \rho(\vec{m}, \vec{m}) R^{\dagger} \otimes$ $R^{\dagger}$ where $R$ is the corresponding element of $S U(2)$, we have

$$
a_{R(\vec{n})}=R \otimes R a_{\vec{n}} R^{\dagger} \otimes R^{\dagger} .
$$

We can also without loss of generality suppose that the measurement is symmetric with respect to interchanges the two spins. This implies that

$$
a_{\vec{n}}=w I+\vec{x} \cdot(\vec{\sigma} \otimes I+I \otimes \vec{\sigma})+\sum_{i, j} z_{i j} \sigma_{i} \otimes \sigma_{j}
$$

with $z_{i j}$ a symmetric matrix.

The covariance condition (11) implies a considerable simplification on the coefficients $w, \vec{x}, z_{i j}$ in (12). Consider the POVM element $a_{\vec{z}}$ corresponding to guessing the spins are polarized along the $+z$ direction. Let $R_{\phi, z}$ be a rotation of angle $\phi$ around the $z$ axis. We have $a_{\vec{z}}=R_{\phi, z} \otimes R_{\phi, z} a_{\vec{z}} R_{\phi, z}^{\dagger} \otimes R_{\phi, z}^{\dagger}$ for all $\phi$. Using (12), this implies that $a_{\vec{z}}$ has the form

$$
\begin{aligned}
a_{\vec{z}}= & w I+\alpha\left(\sigma_{z} \otimes I+I \otimes \sigma_{z}\right)+\beta \sigma_{z} \otimes \sigma_{z} \\
& +\gamma\left(\sigma_{x} \otimes \sigma_{x}+\sigma_{y} \otimes \sigma_{y}\right)
\end{aligned}
$$

where $\alpha, \beta, \gamma$ are three real numbers.

A final simplification results if we recall that the operators $a_{\vec{n}}$ must sum to the identity:

$$
\int d \vec{n} a_{\vec{n}}=\int_{S U(2)} d R R \otimes R a_{+\vec{z}} R^{\dagger} \otimes R^{\dagger}=I .
$$

Using (13) this implies that

$$
\begin{aligned}
a_{\vec{z}}= & I+\alpha\left(\sigma_{z} \otimes I+I \otimes \sigma_{z}\right) \\
& +\gamma\left(2 \sigma_{z} \otimes \sigma_{z}-\sigma_{x} \otimes \sigma_{x}-\sigma_{y} \otimes \sigma_{y}\right)
\end{aligned}
$$

which only depends on two parameters $\alpha$ and $\gamma$.

It is now easy to compute the restriction on the two parameters $\alpha$ and $\gamma$ which result from each of the three positivity conditions enumerated above:

$$
\begin{aligned}
& a_{\vec{n}} \geq 0 \Rightarrow \gamma \leq 1,1+\alpha+\gamma / 2 \geq 0, \\
& 1-\alpha+\gamma / 2 \geq 0 \\
& a_{\vec{n}}{ }^{2} \geq 0, \Rightarrow \gamma \leq 2,1+\gamma-\alpha^{2} \geq 0, \\
& a_{\vec{n}} \text { and } a_{\vec{n}}{ }^{2} \geq 0 \Rightarrow \gamma \leq 1,1+\gamma-\alpha^{2} \geq 0 .
\end{aligned}
$$

These constitute convex sets. The extremal points of these convex sets will be the optimal measurements. To understand what extremal point corresponds to what optimal measurement we introduce a fidelity function $f$. We now study different fidelity functions.

The covariance of the measurement set up implies that $f$ is a function only of the angle between the direction in which the spins are polarized $\vec{m}$ and the direction guessed by the POVM element $a_{\vec{n}}$. Thus in the case of outcome $+z, f$ is a function of $m_{z}$ only. It is convenient to expand $f$ in Legendre polynomials

$$
\begin{aligned}
f(+z, \vec{m}) & =\sum_{n=0}^{\infty} f_{n} P_{n}\left(m_{z}\right) \\
& =f_{0}+f_{1} m_{z}+f_{2} \frac{3 m_{z}^{2}-1}{2}+\cdots .
\end{aligned}
$$

To compute the average fidelity we need the probability of each outcome. Suppose that the spins point in direction $\vec{m}$ and that the measurement outcome is $+z$. This occurs with probability

$$
P(+z \mid \vec{m})=\operatorname{Tr} \rho(\vec{m}, \vec{m}) a_{\vec{z}}=1+\alpha m_{z}+\frac{\gamma}{2} \frac{3 m_{z}^{2}-1}{2} .
$$

The average fidelity is therefore

$$
\begin{aligned}
F & =\int_{-1}^{+1} \frac{d m_{z}}{2} \int_{0}^{2 \pi} \frac{d \phi}{2 \pi} f(+z, \vec{m}) P(+z \mid \vec{m}) \\
& =f_{0}+\frac{\alpha}{3} f_{1}+\frac{\gamma}{10} f_{2} .
\end{aligned}
$$

Thus only the first three coefficients enter into the average fidelity. (In the case of covariant measurements on $N$ parallel spins only the $N+1$ first coefficients of the expansion of $f$ will enter into the average fidelity).

Using eqs. (16, 17, 18) and (21) it is straightforward to find the optimal measurement for an arbitrary fidelity function in the case of parallel spins, antiparallel spins and LOCC measurements. As a first illustration, let us consider the example studied in [2] and [3] in which the fidelity has the form $f(\vec{n} \mid \vec{m})=\left|\left\langle\uparrow_{\vec{m}} \mid \uparrow_{\vec{n}}\right\rangle\right|^{2}=(1+\cos \theta) / 2$. Thus in this example $f_{0}=1 / 2, f_{1}=1 / 2, f_{2}=0$ and therefore $F=1 / 2+\alpha / 6$. In this case the largest fidelity is obtained by taking for $\alpha$ the largest possible value. In the case of two parallel spins the largest possible value of $\alpha$ is $\alpha_{\max }=3 / 2$ corresponding to $F_{\|}=3 / 4=0.75$, a result already obtained in [2] and [3]. In the case of two antiparallel spins $\alpha_{\max }=\sqrt{3}$ corresponding to $F_{\perp}=1 / 2+1 /(2 \sqrt{3}) \simeq 0.788$, a result already obtained in [10]. In the case of measurements carried out using only LOCC, $\alpha_{\max }=\sqrt{2}$ corresponding to $F_{L O C C}=1 / 2+1 /(3 \sqrt{2}) \simeq 0.736$. Thus for this fidelity collective measurements on antiparallel spins are better than collective measurements on parallel spins which are themselves better than LOCC measurements on parallel (or antiparallel) spins.

Note that if the spins are parallel or antiparallel, optimal measurements that use a 1 dimensional ancilla 
(which could be the singlet state) have been exhibited in [3] and 10. In the case of LOCC measurements it is easy to check that a simple optimal strategy consists in Alice making a von Neumann measurement of spin along some direction $\vec{a}$ and Bob making a von Neumann measurement of spin along an orthogonal direction $\vec{b}(\vec{a} \cdot \vec{b}=0)$. Denote by $\vec{\alpha}= \pm \vec{a}$ and $\vec{\beta}= \pm \vec{b}$ the results of the two measurements. Then the guessed direction is the bisectrix $\vec{\alpha}+\vec{\beta}$ of the two results. Thus in all cases the optimal measurements can be implemented using rather simple strategies which necessitate low dimensional ancillas. This should be compared with the covariant measurements which, although useful for the theoretical analysis, require infinite dimensional ancillas.

As a second illustration consider the case where the fidelity is $f=\sin ^{2} \theta=1-\cos ^{2} \theta$. In this case $f_{0}=2 / 3$, $f_{1}=0, f_{2}=-2 / 3$, hence $F=2 / 3-\gamma / 15$ and the best measurement is that which has the smallest value of $\gamma$. In the case of collective measurements on parallel spins the smallest value is $\gamma_{\min }=-2$ yielding a fidelity $F_{\|}=4 / 5=0.8$. For collective measurements on antiparallel spins or LOCC measurements the minimum value is $\gamma_{\min }=-1$ yielding an optimal fidelity $F_{\perp, L O C C}=11 / 15 \simeq 0.733$. In this case measurements on two parallel spins are better than measurements on antiparallel spins or LOCC measurements which are both equivalent. Thus for some fidelities measurements on parallel spins are better, for other fidelities measurements on antiparallel spins are better, and in all cases LOCC measurements are the worst.

In conclusion the present article gives explicitly the optimal measurements and optimal fidelity for all possible fidelity functions in the cases of parallel spins, antiparallel spins, and LOCC measurements. This provides an interesting target for future experiments since it provides a criterion for putting "non locality without entanglement" into evidence.

I would like to thank N. Gisin, N. Linden and S. Popescu for stimulating and helpful discussions, and Dietmar Fisher for pointing out his recent work to me. I am a research associate of the Belgian National Fund for Scientific Research.

[1] C. W. Helstrom, Quantum Detection and Estimation Theory, Academic, New York, 1976.

[2] A. S. Holevo, Probabilistic and Statistical Aspects of Quantum Theory, North Holland, Amsterdam, 1982.

[3] S. Massar and S. Popescu, Phys. Rev. Lett. 74 (1995) 1259.

[4] R. Derka, V. Buzek, A.K. Ekert, Phys. Rev. Lett. 80
(1998) 1571

[5] J.I. Latorre, P. Pascual, R. Tarrach, Phys.Rev. Lett. 81 (1998) 1351

[6] A. Peres and W. K. Wootters, Phys. Rev. Lett. 66, 1119 (1991)

[7] C. H. Bennett, D. P. DiVincenzo, C. A. Fuchs, T. Mor, E. Rains, P. W. Shor, J. A. Smolin, and W. K. Wootters, Phys. Rev. A 59 (1999) 1070

[8] R. Gill and S. Massar, Phys. Rev. A 61, 042312 (2000)

[9] A. Peres, Phys. Rev. Lett. 77 (1996) 1413

[10] N. Gisin and S. Popescu, Phys. Rev. Lett. 83 (1999) 432

[11] Dietmar Fisher, private conversation; see also D.G. Fischer, S.H. Kienle, and M. Freyberger, Phys. Rev. A 61, 032306 (2000) 